\title{
Recent advances in the management of epilepsy
}

\author{
F Islam', S Ahmed ${ }^{2}$, B H N Yasmeen ${ }^{3}$, N Begum ${ }^{4}$, S Sharmin ${ }^{5}$, L Helaly ${ }^{6}$, R T Hyder ${ }^{7}$
}

1 Dr. Farooqul Islam

Assistant Professor

Dr. Shirajul Islam Medical College

${ }^{2}$ Dr. Shafi Ahmed

Assistant Professor

Dept. of Paediatrics

Nortern International Medical

College, Dhaka

3 Dr. B H Nazma Yasmeen

Associate Professor

Dept of Pediatrics

Nortern International Medical

College, Dhaka

${ }^{4}$ Dr. Nasreen Begum

Assistant Professor

Nortern International Medical

College, Dhaka

${ }^{5}$ Dr. Shabnam Sharmin

Lecturer Physiology

Shaheed Suhrawardi Medical College

${ }^{6}$ Dr. Laila Hilaly

Consultant

Bangladesh Thalassaemia Hospital Dhaka

${ }^{7}$ Dr. Rumy Tabrez Hyder

Assistant Professor

Dept. of Paed

MH Samorita Medical College, Dhaka

\section{Correspondence}

Dr. Farooqul Islam

Assistant Professor

Dr. Shirajul Islam Medical College
Abstract

Epilepsy is a complex disorder affecting brain function having a variety of contributing factors. The genetic predisposition plays a key role in the genesis of epilepsy. Antiepileptic drugs (AEDs) provide effective control in majority of patients with different seizure types. But some refractory cases and those who cannot tolerate the conventional AEDs, it is difficult to control the seizures adequately. On the basis of different mechanisms of action and considering the side effects of drugs, various newer approaches have been evolved during the recent years in the rational management of epilepsy. A brief account of these newer treatment modalities has been focused and incorporated in this review in order to enlighten the readers with the possible beneficial effect of this regimen and their limitations as well.

Keywords : Antiepileptic drugs, defective genes, neurotransmitter, newer approaches etc.

\section{Introduction}

Epilepsy is not a single disorder rather it is a group of disorders of central nervous system characterized by paroxysmal cerebral dysrythmia and manifested by brief episodes of seizures with or without loss or disturbance of consciousness ${ }^{1}$. When a person has two or more seizures, he is considered to be epileptic $^{1}$. It is a common neurological disorder affecting about $0.5 \%$ of the population in which cluster of neurons sometimes signal abnormally ${ }^{2}$. During a seizure, neurons fire as many as 500 times per second, compared to the normal rate of about 80 times per second ${ }^{2}$.

Hypotheses in vogue for genesis of epilepsy ${ }^{1,2}$

1. Abnormal CNS wiring: this may occur while development of brain leading to abnormal neuronal connections.

2. Neurotransmitter imbalance: The spread of seizure activity may be mediated either by increase of excitatory neurotransmitters (mainly glutamate) or by decrease of inhibitory neurotransmitters like GABA (Gammaaminobutyric acid) and possibly glycine.

3. Disruption of peri-neuronal cell membrane: important for molecules to move in and out of membranes and how cell nourishes and repair the membrane.

4. Brain derived neurotrophic factor: activates receptors in the hippocampus during epileptogenesis.

\section{Causes of epilepsy}

About $40 \%$ of patients with epilepsy have a genetic component towards it aetiology ${ }^{3}$.

I. Genetic factors: More than 500 genes are involved in epilepsy ${ }^{3}$ and people's susceptibility to seizures may be triggered by following factors:

1) defective gene for ion channels

2) missing of cystatin B protein gene causing progressive myoclonus epilepsy

3) missing of a carbohydrate breaking gene that causes Lafora body disease

4) gene mediating resistance of antiepileptic drugs (AEDs).

II. Various disorders: like brain tumors, alcoholism, Alzheimer's disease, stroke, meningitis, hydrocephalus, cerebral palsy may precipitate epilepsy ${ }^{4}$. About $32 \%$ of all cases of newly developed epilepsy in elderly people are due to cerebrovascular disease.

III. Head injury: Head injury constitutes an important contributing factor for epilepsy.

IV. Pre and perinatal insult: About $2.0 \%$ of seizures in children are due to prenatal insult, perinatal asphyxia, cerebral palsy or other neurological abnormalities ${ }^{4}$.

\section{Treatment modalities}

Currently available treatment controls about $80 \%$ of seizures by drugs and/or surgery and $20 \%$ seizures remain intractable 4 .

Table-I. Classification of antiepileptic drugs (AEDs)

\begin{tabular}{|l|l|l|}
\hline 1 & Barbiturates & $\begin{array}{l}\text { Phenobarbitone, Mephobarbitone, } \\
\text { Primidone }\end{array}$ \\
\hline 2 & Hydantoin & Phenytoin \\
\hline 3 & Iminostilbene & Carbamazepine \\
\hline 4 & Succinimide: & Ethosuximide \\
\hline 5 & $\begin{array}{l}\text { Aliphatic } \\
\text { carboxylic acid }\end{array}$ & Valproic acid \\
\hline 6 & Benzodiazepines & Clonazepam, Clobazam, Diazepam \\
\hline 7 & Newer drugs & $\begin{array}{l}\text { Lamotrigine, Gabapentin, Vigabatrin, } \\
\text { Felbamate, Topiramate, Tiagabine, } \\
\text { Zonisa mide, Levitiracetam }\end{array}$ \\
\hline 8 & Miscellaneous & Trimethadione, Phenacimide. \\
\hline
\end{tabular}


It was an assumption for long period that a single drug would be enough for treating epilepsy. Bromide was the first drug introduced in 1857 for the control of epilepsy. Later in 1912 Phenobarbital was discovered as an important member of AED family and then in 1938 Phenytoin, structurally related drug to barbiturate, was discovered. All these drugs (except Bromide) are being used very commonly to provide effective seizure control and phenytoin still plays a major role in the management of epilepsy ${ }^{5}$. Later on, the introduction of carbamazepine and lamotrigine has opened up new avenues in the management of epilepsy. The latest surge of newer AEDs during the last decade has seen the emergence of drugs like gabapentin, vigabatrin, topiramate, tiagabine, oxycarbazepine, zonisamide and levitiracetam ${ }^{6}$.

\section{General principles of management of Epilepsy}

Early initiation of treatment with AEDs, following recurrent seizures, almost always proves to be effective and improves prognosis. Initially one drug regimen should be started, unless status epilepticus exists, to achieve therapeutic benefit with careful monitoring of the dosage and saliva or plasma concentration of drugs, if possible. To minimize dose related adverse effects, addition of a second drug is advisable when the first drug fails to control the seizures. The multi-drug therapy should be started at a reduced dose of each drug with careful monitoring. It should be remembered while selecting the multi-drug regimen that the combination acts by well defined mechanisms like: either by promoting $\mathrm{Na}+$ channel activation and /or by enhancing GABA mediated synaptic inhibition. Alternative line of approach should also continue till the seizure is controlled.

Abrupt withdrawal of any AED should be discouraged (esp. barbiturates and benzodiazepines) as they may precipitate status epilepticus. If a patient is seizure free for 3-4 years, gradual withdrawal of drugs (over a period of 3-6 months) is advocated. To increase patient compliance, drugs are best administered in single or twice daily doses. Many AEDs are quite long acting with a relatively low plasma clearance and greater half life ( $>12$ hrs.). The therapeutic index being low, these drugs exhibit toxicity. Dose increments are made gradually at two-weekly intervals to achieve the minimum effective dose.

Routine monitoring of plasma and/or saliva drug concentration with concomitant renal or hepatic evaluation is mandatory in old age, during pregnancy or especially when sodium valproate or phenytoin is used. A small increment in the dose of phenytoin may lead to toxicity due to its dose dependent kinetics (zero order kinetics). The therapeutic efficacy of a drug and/or the appearance of toxicity should be assessed by monitoring the plasma and saliva drug concentrations.

Effective plasma levels of some of the commonly used AEDs ${ }^{7}$ have been given below:

Table II: Effective plasma level of antiepileptic drugs

\begin{tabular}{|l|l|l|c|}
\hline Drugs & $\begin{array}{l}\text { Effective } \\
\text { level } \\
(1 / 4 \mathrm{~g} / \mathrm{ml})\end{array}$ & $\begin{array}{l}\text { High } \\
\text { effective } \\
\text { level }(1 / 4 \mathrm{~g} / \mathrm{ml})\end{array}$ & $\begin{array}{l}\text { Toxic level } \\
(1 / 4 \mathrm{~g} / \mathrm{ml})\end{array}$ \\
\hline Carbamazepine & $4-12$ & 7 & $>8$ \\
\hline Primidone & $5-15$ & 10 & $<12$ \\
\hline Phenytoin & $10-20$ & 18 & $>20$ \\
\hline Phenobarbital & $10-40$ & 35 & $>40$ \\
\hline Ethosuximide & $50-100$ & 80 & $>100$ \\
\hline Valproate & $50-100$ & 80 & $>100$ \\
\hline
\end{tabular}

\section{Pregnancy, lactation and AEDs}

Pregnancy and epilepsy can affect each other and at times become harmful to the developing foetus due to anoxia and metabolic disorder. Folic acid supplement should be advised to patients on AEDs (phenobarbital and phenytoin) - deficiency of which may cause neural tube defects. Similarly, pregnant mothers on AED therapy should be given prophylactic oral vitamin $\mathrm{K} 1$ supplement $(10 \mathrm{mg} /$ day) during the last few weeks of pregnancy to avoid the danger of postpartum haemorrhage and neonatal intracerabral haemorrhage. Almost all the AEDs

(carbamazepine being the safest) cross the placental barrier and are excreted in breast milk thereby causing sedation and poor sucking by the breast fed baby ${ }^{2}$. Increased frequency of malformation at birth (known as anticonvulsant embryopathy) is another problem in mothers taking AEDS especially phenytoin or phenobarbital. In children suffering from epilepsy, a single drug regimen with minimal doses should be initiated to avoid complications. Febrile convulsions in children do not necessarily always precipitate epilepsy and should be treated early with paracetamol and diazepam. Prolonged use of phenytoin or phenobarbital may affect the cognitive development of the child.

\section{Treatment of status epilepticus}

- Status epilepticus (SE) is a neurological emergency where rapid seizure control is mandatory because the longer the episode of SE remains untreated, most of the drugs become refractory and the control becomes more difficult resulting in possible permanent brain damage.

- Intravenous lorazepam (4 mg) is now the preferred initial choice. It may be repeated after 10 minutes, if necessary.

- Alternatively, Clonazepam $1 \mathrm{mg}$ i.v. or Diazepam 10-20 mg i.v. may be tried. Since Diazepam is more likely to cause hypotension and respiratory depression and its effect is short lasting (about 20 minutes), either Phenytoin (15-18 mg / kg i.v.) or Phenobarbitone (10-20 mg/kg i.v.) may be considered.

- In refractory cases, Thiopental or Profol or Midazolam may be preferred with full intensive care support system.

- Magnesium sulphate (4 gm i. v.) infusion for $24 \mathrm{hrs}$ after the last seizure may be a better option than either Phenytoin or Diazepam for the treatment of seizure disorder of eclampsia ${ }^{8}$.

- Some of the AEDS (Phenytoin, Carbamazepine, Barbiturates, Topiramate, Oxcarbazepine) may cause contraceptive failure (when used with oral contraceptive pills) by inducing hepatic microsomal enzymes. In such cases, the dose of oestrogen can be increased (at least upto $50 \mu \mathrm{g} /$ day) to achieve reasonably satisfactory contraception. However, drugs like sodium valproate and lamotrigine do not cause enzyme induction and contraceptive failure.

\section{Newer antiepileptic drugs (AEDs)}

A brief account of newer antiepileptic drugs has been outlined below:

1. Lamotrigine: Effective as monotherapy or add-on therapy in partial and secondarily generalized tonic-clonic seizures in adults and LennoxGastaut syndrome (characterized by a multiple seizure type disorder of childhood with mental retardation and refractory to anti-seizure medication). It is also effective in juvenile myoclonic as well as absence seizures. 
It has a broader spectrum of antiseizure activity, possibly by inhibiting synaptic release of glutamate by acting at $\mathrm{Na}+$ channel themselves. Completely absorbed orally, plasma half-life between 24 to 35 hrs and can be used with once daily dose ranging from $100-300 \mathrm{mg}$. Metabolised primarily by glucuronidation. Addition of valproate markedly increases plasma concentration of lamotrigine.

Adverse effects include drowsiness, dizziness, diplopia, headache and hypersensitivity reaction.

2. Gabapentin : It is effective in partial as well as grandmal epilepsy. It is a GABA analogue which increases its release by inhibiting GABA uptake. It is fairly well absorbed orally, half life ranges between 5-8 hrs, administered 2-3 times a day and total dose not exceeding $2.4 \mathrm{gm} /$ day. Adverse effects include tremors, ataxia, drowsiness and dizziness.

3. Vigabatrin : It is useful in the treatment of partial and to some extent in grandmal \& infantile seizures. But it worsens myoclonic or absence seizures. It prevents inactivation of GABA by inhibiting GABA aminotranferase. It is well absorbed orally and its half life is 6-8 hrs. It is administered in twice daily dosage form of $500 \mathrm{mg}$ each. Dose may be increased up to 2-3 gm/day. Adverse effects include drowsiness, dizziness, weight gain, agitation, mental confusion and psychosis. This drug should not be used in pre-existing mental disease. Visual defects (irreversible tunnel vision) have been reported on long term use of this drug.

4. Felbamate : It is effective mainly in partial seizures. It is believed to block NMDA receptors via glycine binding sites. It is absorbed orally and has a long half life ( $20 \mathrm{hrs}$ ). It increases plasma level of phenytoin and sodium valproate and decreases that of carbamazepine. The use of this drug is limited as it causes aplastic anaemia and hepatitis.

5. Topiramate : Partial and grandmal seizures are controlled by this drug. This drug is quite useful in infantile spasm also. It also has some effect on absence seizures. This drug has multiple actions including blockade of voltage-gated sodium channels, potentiation of GABA activity and inhibition of Kainite (KA) effect on AMPA (alpha- amino-3 hydroxy-5 methylisoxazole- 4-propionate) receptors; both KA and AMPA receptors are activated to cause excitation of CNS where glutamate is the principle transmitter ${ }^{7}$. Therapeutic spectrum of Topiramate resembles Phenytoin with fewer side effects. It has been reported to be teratogenic in animals. So it is contraindicated in women of child bearing age. It is effective both in adults and children with refractory partial seizures with or without secondary generalized tonic- clonic seizures in patients with Lennox- Gastaut syndrome. This drug is rapidly absorbed when used orally and its half-life is between 20-30 hrs. It is administered in a dose range of 200-600 mg/day. Common adverse effects include drowsiness, dizziness, mental clouding, anxiety, cognitive slowing and urolithiasis.

6. Tiagabine : A derivative of nipecotic acid, tiagabine is used as an add on drug for partial seizures which act by inhibiting neuronal and glial uptake of GABA. It is very well absorbed orally with nearly $100 \%$ bioavailability. Its half-life is $5-8 \mathrm{hrs}$ and it is used in dosage ranging from 16 to $56 \mathrm{mg}$ four times a day. It is well tolerated with minor doserelated adverse effects like nervousness, dizziness, drowsiness, tremors and depression. Psychosis or ataxia may require withdrawal of the drug.

7. Zonisamide : It is a sulphonamide derivative. It acts on the sodium channel as well as on the voltage- dependent calcium channels. It is effective on different types of seizures like partial, grandmal, infantile and myoclonus. It is well absorbed orally with a long half-life of 1-3 days. It is used in dosage ranging from $100-600 \mathrm{mg} /$ day. Adverse effects include drowsiness, ataxia, cognitive impairment and skin rash.

8. Levetiracetam : It is a piracetam analogue whose mode of action is unclear. Probably it acts through modulation of GABA receptors and also through $\mathrm{Ca} 2+$ and $\mathrm{K}+$ channels. It is mainly used for the treatment of partial seizures. It also has a potentially broader spectrum of use. It is completely absorbed after oral administration with a half-life of 6-8 hrs. Dosage ranges from 0.5 to $1 \mathrm{gm}$ twice daily. Drowsiness, asthenia and somnolence are the adverse effects.

\section{Some other AEDs of potential therapeutic benefit:}

1. Stripentol: derived from alcohol used in partial and absence seizures

2. Retigabine: possibly acts through $\mathrm{K}+$ channels

3. Remacemide10: NMDA receptor modulator

4. Talampanel: AMPA receptor antagonist

5. Pregabalin: related to Gabapentin

6. Delivering drugs directly to the part of the brain from where seizures originate e.g. sodium valproate in fatty capsule.

7. Transporter proteins: many drugs need these to get into the brain.

8. Melatonin: might reduce seizures in some children.

\section{Newer approaches in the management of epilepsy}

A. Diet : unusual diet like "Ketogenic diet" that helps body to break down fat instead of carbohydrate.

\section{B. Vagus nerve stimulation (VNS) ${ }^{11}$}

VNS was approved by US FDA in 1997 for epileptic patients (older than 12 years of age) not controlled by standard medication. It is a batterypowered device, surgically implanted under the skin of chest and attached to vagus nerve in lower part of the neck. It delivers short bursts of electrical energy to brain via vagus nerve. On an average, it reduces short term seizure frequency by $20-40 \%$ with an approximately long term decrease in mean seizure frequency of $40-50 \%$ and the dose of the antiepileptic medication can be further reduced in those patients who cannot tolerate these drugs. Patients who suffer from complex partial seizures or generalized seizures with loss of consciousness not responding to standard anticonvulsant medication and patients who cannot undergo brain surgery are considered good candidates for VNS. It also may be recommended as a treatment for photosensitive epilepsy and epilepsy resulting from head injury. ${ }^{12}$

Investigators have suggested that VNS possibly increases seizure threshold by causing widespread release of GABA and glycine in the brain $^{11 .}$ Adverse effects like hoarseness, voice change, difficulty in swallowing, cough or dyspnoea are mild which appears during stimulation but diminishes over time. About the suitability of VNS in the management of epilepsy, it may be described as a long-lasting, hassle free and on-demand therapy, with no interactions and potential life threatening adverse effects. Moreover, the reduction of depressive symptoms by $\mathrm{VNS}^{13}$ and its mood stabilizing effect appears to be an added advantage.

Finally, the continued search and discovery of newer antiepileptic drugs during the last decade has not only failed to revolutionize the approach 
of the management of a complex disorder like epilepsy, but also none of these agents has been shown to be superior to the standard drugs like phenytoin, carbamazepine or sodium valproate. An estimated $1 \%$ of the general population has epilepsy and close to $30 \%$ of these patients, is intractable to medications ${ }^{12}$; many others have their seizures controlled at the expense of unacceptable adverse effects from pharmacotherapy.

\section{Surgery}

All patients with refractory epilepsy are not the candidates for surgery. Child patients with intractable epilepsy may have a progressive disorder that is medically, physically and socially disabling. Surgical resection of the epileptogenic zone or lesional pathology, or both, may significantly reduce seizure tendency in selected patients ${ }^{14}$. Favourable candidates for focal cortical resection include individuals with medical temporal lobe epilepsy and partial seizures related to primary brain tumor or vascular anomalies ${ }^{14 .}$ The objective of pre-surgical investigations, dominated by scalp-recorded video electroencephalography and magnetic resonance imaging is the localization of the epileptogenic focus.

Reports suggest that initiation of surgical treatment at an early stage significantly improves the quality of life, seizure free duration and acceptable socialization ${ }^{15}$.

\section{Conclusion}

In the search of an ideal antiepileptic drug that could suppress all types of seizures without causing much adverse effects, more recently introduced drugs have some advantage. ${ }^{16}$ But the hallmark of success in the management of epilepsy is patient compliance and rigidly advocated regularity of medication with full patient cooperation.

\section{References}

1. Gardiner RM, Lehesjoki AE. Genetics of the epilepsies. Curr Opin neurol 2000; 13:157-164

2. Bhattacharya SK, Sen P, Ray A. Motor disorders, In: Das Prasun K's Pharmacology. 2nd Ed. New Delhi: Elsevier, 2004:247-252.

3. Gardiner RM. Genetics of epilepsies. In: Sinha KK, Chandra P's Advances in Neurosciences. Vol -2 , Kolkata: Association of Neuroscientists of Eastern India, 2001:155- 176.

4. Gangopadhyay PK, Biswas A, Senapati AK and Gangopadhyay G. Genetics of Epilepsies. Jour Ind Med Assoc. (JIMA) 2004,102 (02):71-72.

5. Bennett PN, Brown MJ. Epilepsy, Parkinsonism and allied conditions. In Clinical Pharmacology. Churchill Livingstone, 9th ed. chapter 20, 2003:413-422.

6. McNamara JO. Drugs effective in therapy of epilepsies. In: Goodman and Gilmans, The Pharamcological basis of therapeutics, McGrawHill, 10th ed; chapter 21, 2001; 521- 547.

7. Katzung BG. Antiseizure drugs. In Basic \& Clinical Pharmacology, McGraws Hill, 9th ed. Chapter 24, 2004:379-400.

8. Eclampsia Trial Collaborative group. Lancet 1995; 345:1455-2463

9. Wilmore LJ. Lamotrigine. Expert review of Neurotherapeutics. 2001; 1:33.

10. Marson AG et al. Levetiracetam, oxcarbazepine, remacemide and zonisamide for drug resistant localization- related epilepsy: a systemic review. Epilepsy Res 2001; 46:259.

11. Ben-Menachem E. Vagus nerve stimulation for the treatment of epilepsy. The Lancet- Neurology 2002; 1:477.
12. Zabara J. Inhibition of experimental seizures in canines by repetitive vagal stimulation. Epilepsia 1992; 33: 1005-1012.

13. Harden CL, Pulver MC, Nikolov B, Halper JP and Labar DR. Effect of Vagus Nerve Stimulation on Mood in Adult EpilepsyPatients. Neurology 1999; 52(supp.2):A238P03122 (Abstract).

14. Cascino GD. Surgical treatment for epilepsy. Epilepsy Res. 2004; 60 (2-3): 179-186.

15. Delalande 0 , Fohlen $M$, Bulteau $C$, Jalin C. Surgery for intractable focal epilepsy in children. Rev; Neurol 2004; 160:195-202.

16. Wallace SJ. Newer antiepileptic drugs: advantages and disadvantages. Brain Dev 2001; 23:277. 